Mathematical Research Letters 2, 751-770 (1995)

\title{
THE K-PROPERTY OF HAMILTONIAN SYSTEMS WITH RESTRICTED HARD BALL INTERACTIONS
}

\author{
NÁNDOR SimÁNYI AND Domokos SzÁsz
}

\section{Introduction}

In 1985, Chernov and Sinai suggested the study of a mechanical model of elastic hard balls, the so-called pencase, which we will call the ChernovSinai pencase (see the manuscript $[\mathrm{S}-\mathrm{Ch}(85)]$ ). Here large hard ball particles move on a torus, elongated in one spatial dimension; since then the cyclic order of the particles remains invariant, each ball can only interact with its two neighbours. Their idea is the following:

On the one hand, because of the restricted form of the interactions, the expected ergodicity of the model could be easier to establish than that of general hard ball systems on tori. On the other hand, this quasi-onedimensional model seems to be a quite suitable mechanical one for the study of the hydrodynamic limit transition (see [Sin(85)]).

For systems of elastic hard balls on a torus Yakov Sinai, in 1963, [Sin(63)] gave a mathematically rigorous version of Boltzmann's celebrated ergodic hypothesis: the system of an arbitrarily fixed number $N$ of elastic hard balls moving in the $\nu$-torus $\mathbb{T}^{\nu}(\nu \geq 2)$ is ergodic (of course, on the submanifold of the phase space specified by the trivial conservation laws).

The aforementioned hypothesis, which we call the Boltzmann-Sinai Ergodic Hypothesis, was first established for the case $N=\nu=2$ in Sinai's celebrated paper $[\operatorname{Sin}(70)]$. After several steps $([\mathrm{S}-\mathrm{Ch}(87)]$ : the case $N=2$, $\nu \geq 2 ;[\mathrm{K}-\mathrm{S}-\mathrm{Sz}(91)]$ : the case $N=3, \nu \geq 2 ;[\mathrm{K}-\mathrm{S}-\mathrm{Sz}(92)]$ : the case $N=4$, $\nu \geq 3$ ) the most general result so far was obtained by Simányi [Sim(92)], who could verify the conjecture for the case $\nu \geq N \geq 2$ (a more detailed survey of the history of Boltzmann's ergodic hypothesis can be found in $[\mathrm{Sz}(95)])$.

The aim of the present work is to verify the $K$-property of models closely related to the mechanical system of hard balls. The restriction we make

Received September 1, 1995.

Research supported by the Hungarian National Foundation for Scientific Research, grant No. 1902. 
is on the graph of interactions. We first assume that it is a tree (i.e., a connected graph without loop) whose edge degree is bounded from above by a constant $D$.

Remarks.

(i) The graph of interactions means that only pairs of particles connected by an edge in this graph can interact (in our model they do this through a hard ball collision) whereas non-connected pairs do not interact at all.

(ii) The degree of an edge of a graph is the number of edges adjacent to the given edge, and then the edge degree of a graph is the maximum of the degrees of all edges.

The precise description of our model will be given soon. Then we prove that such a system is a $K$-mixing one if $\nu \geq D+2$, and, moreover, for the case $\nu=D+1$ we obtain that none of the relevant Lyapunov exponents vanishes and the ergodic components are open.

By strengthening the methods used in treating the aforementioned tree interactions we can also treat an interaction graph which is not a tree: a circle of length $N$. This result implies the $K$-property of the Chernov-Sinai pencase in dimension $\nu=4$. It is easy to see that in the Chernov-Sinai pencase, in dimensions $\nu \leq 4$, the graph of interactions is a simple cyclic one (i.e., the graph that consists of just one circle of length $N$ ). Our methods also imply that the Chernov-Sinai pencase has nonzero Lyapunov exponents and open ergodic components in our physical space dimension $\nu=3$. (The pencase as a system of ball particles loses order invariance if $\nu \geq 5$, and if one wants to maintain this property, systems of particles with other, less natural shapes can, in fact, be introduced and treated by our methods.) It is worth mentioning that in the low dimensional cases (starting from $\nu=4$ ) our results provide the first mechanical models where the ergodicity of the system is established for an arbitrary number of degrees of freedom (apart from a model introduced in [B-L-P-S(92)], where the boundary of the vessel also contributes to the hyperbolic effects).

Furthermore, an important advantage of the hereby presented method for going down to physical dimensions is that it avoids the use of more involved algebraic tools that seem unavoidable in the case of treating hard sphere systems in the general framework.

We now introduce the model and formulate our results. Assume that, in general, a system of $N(\geq 2)$ balls, identified as $1,2, \ldots, N$ are given in $\mathbb{T}^{\nu}$, the $\nu$-dimensional unit torus $(\nu \geq 2)$. Denote the phase point of the $i$ th ball by $\left(q_{i}, v_{i}\right) \in \mathbb{T}^{\nu} \times \mathbb{R}^{\nu}$. Assume also that the graph $\mathcal{G}:=(\mathcal{V}, \mathcal{E})$ of interactions is given, where the set of edges $\mathcal{E}$ makes a non-oriented tree (i.e., 
a connected graph without a loop) on the set of vertices $\mathcal{V}:=\{1,2, \ldots, N\}$. (In the case of the mechanical model of $N$ hard balls on $\mathbb{T}^{\nu}$ this graph is, of course, the complete one.) Further, for every $\{i, j\}: 1 \leq i, j \leq N, i \neq j$, the potential functions $U_{i, j}: \mathbb{R}_{+} \rightarrow \mathbb{R}_{+} \cup\{\infty\}$ are given as follows

$$
U_{i, j}(r)= \begin{cases}\infty, & 0 \leq r<d_{i, j} \\ 0, & r \geq d_{i, j}\end{cases}
$$

where the numbers $d_{i, j}=d_{j, i}$ are nonnegative. They determine the set of edges $\mathcal{E}:\{i, j\} \in \mathcal{E}$ if and only if $d_{i, j}>0$. Moreover, the interaction between the pair $\{i, j\}$ of particles with configuration coordinates $q_{i}, q_{j} \in \mathbb{T}^{\nu}$ is

$$
\tilde{U}_{i, j}\left(q_{i}, q_{j}\right)=U_{i, j}\left(\left\|q_{i}-q_{j}\right\|\right) .
$$

where $\|$.$\| denotes the euclidean distance in the torus \mathbb{T}^{\nu}$. Then the standard billiard ball flow $\left(\mathbf{M}, S^{\mathbb{R}}, \mu\right)$ with the hard core $\mathcal{D}$-interaction, where $\mathcal{D}=$ $\left\{d_{i, j}: 1 \leq i<j \leq N\right\}$, is then defined similarly to the definition of the model in, for instance, Section 1 of $[\mathrm{K}-\mathrm{S}-\mathrm{Sz}(92)]$. We adopt the notations of that paper for the basic ingredients of the flow.

The first main result of our paper is the following:

Theorem A. Assume that the edge degree of the tree of interactions is $D$, and for some $N \geq 2, \nu \geq D+2$, Int $\mathbf{Q}$ (or, equivalently, Int $\mathbf{M}$ ) is connected. Then the standard billiard ball system $\left(\mathbf{M},\left\{S^{t}\right\}, \mu\right)$ with a $\mathcal{D}$-interaction is a $K$-flow.

Remarks.

(i) In general, if Int $\mathbf{M}$ decomposes into a finite number of connected components, then our methods provide that the standard billiard ball flow with a $\mathcal{D}$-interaction is a $K$-system on each of these connected components.

(ii) As has been proved in recent manuscripts by N. I. Chernov and C. Haskell, $[\mathrm{Ch}-\mathrm{H}(95)]$ on the one hand, and by D. Ornstein and B. Weiss, $[\mathrm{O}-\mathrm{W}(95)]$ on the other hand, the $K$-mixing property of a semi-dispersing billiard flow actually implies its Bernoulli property as well.

Our second main result concerns the so-called cyclic interaction, where $\mathcal{E}_{c}:=\{\{1,2\},\{2,3\}, \ldots,\{N-1, N\},\{N, 1\}\}$. Let $\mathcal{D}_{c}$ be a corresponding matrix of interaction ranges.

Theorem B. The standard billiard ball system with a cyclic interaction is a K-system if $\nu \geq 4$ and Int $\mathbf{Q}$ is connected.

As mentioned before, an interesting mechanical realization of the cyclic interaction is the Chernov-Sinai pencase. Here the torus is an elongated 
one, i.e., (physical) balls of radii $r$ move on a torus $\mathbb{T}_{L}^{\nu}:=\mathbb{R}^{\nu} /\left(L \mathbb{Z} \times \mathbb{Z}^{\nu-1}\right)$ (in other words, $d_{i, j}=2 r$ ) for every $\{i, j\} \in \mathcal{E}_{c}$. Now, as a particular case of Theorem B, we obtain

Corollary. If $\nu=4$ and $\sqrt{3} / 4<r<1 / 2$, then the Chernov-Sinai pencase is a K-system.

We note that actually the phase space of the pencase consists of exactly $N$ ! connected, isomorphic components whenever $\nu \leq 4$ and $\sqrt{\nu-1} / 4<$ $r<1 / 2$. Thus, of course, the $K$-property can only hold on each of them separately.

By fortifying the inductive arguments we also obtain the following results:

Theorem C. Assume that the edge degree of the graph of interaction is $D$. If $N \geq 2, \nu=D+1$, then, almost everywhere, none of the relevant Lyapunov exponents of the standard billiard ball system $\left(\mathbf{M},\left\{S^{t}\right\}, \mu\right)$ with a $\mathcal{D}$-interaction vanishes. Furthermore, the ergodic components of the system are open (and thus of positive measure), and on each of them, the flow has the $K$-property.

Theorem D. For the models of Theorem B and its Corollary, in dimension $\nu=3$, almost everywhere, none of the relevant Lyapunov exponents vanishes. Furthermore, the ergodic components of the system are open (and thus of positive measure), and on each of them, the flow has the K-property.

General strategy. The basic notion in the theory of semi-dispersing billiards is that of the sufficiency of a phase point or, equivalently, of its orbit. The conceptual importance of sufficiency can be explained as follows:

In a suitably small neighbourhood of a (typical) phase point of a dispersing billiard the system is hyperbolic, i.e., its relevant Lyapunov exponents are not zero. For a semi-dispersing billiard the same property holds for sufficient points only! Physically speaking, a phase point is sufficient if its trajectory encounters in its history all possible degrees of freedom of the system. Then the fundamental theorem of semi-dispersing billiards (see the main result of [S-Ch (87)] ) says that, under certain conditions, a suitably small neighbourhood of a sufficient phase point does belong to one ergodic component. As a consequence, if, for instance, almost every phase point of a semi-dispersing billiard is sufficient, then this property implies that the ergodic components are open and therefore, that their number is countable. To obtain, however, (global) ergodicity of the flow, a more stringent property of the subset of non-sufficient points is needed. For that purpose, in our work with A. Krámli [K-S-Sz (89)], the topological property 
of slimness (earlier misleadingly called 'residuality'), closely related to that of topological codimension 2, was suggested and used in later works, too.

Definition 1.3. We say that a subset of a smooth manifold $M$ is slim if it can be covered by a countable union of codimension 2 (i.e., at least two), closed subsets of $\mu$-measure zero, where $\mu$ is a smooth measure on $M$.

Here the dimension of a separable metric space is its topological or Hurewicz dimension, which is any one of the following equivalent notions: the small inductive, the large inductive, or the covering dimension (see, for instance, [H-W(48)]).

The paper is organized as follows: Section 2 provides the proofs of the theorems and their corollaries assuming Main Lemmas 2.4 and 2.7 of geometric-algebraic nature. Then Main Lemma 2.4 related to tree interactions and used in the proofs of Theorems $\mathrm{A}$ and $\mathrm{C}$ is established in Section 3, while Main Lemma 2.7 related to the cyclic interaction and used in the proofs of Theorems B and D gets verified in Section 4.

\section{Proof of the theorems assuming Main Lemmas 2.4 and 2.7}

Definition 2.1. $\Sigma=\left(\sigma_{1}, \ldots, \sigma_{m}\right)$ is a connected symbolic collision sequence if its collision graph $\mathcal{G}_{\Sigma}:=\left(\mathcal{V}=\{1,2, \ldots, N\}, \mathcal{E}_{\Sigma}:=\left\{\left\{i_{k}, j_{k}\right\}\right.\right.$ : where $\left.\left.\sigma_{k}=\left\{i_{k}, j_{k}\right\}, 1 \leq k \leq m\right\}\right)$ is connected.

Definition 2.2. A symbolic collision sequence $\Sigma=\left(\sigma_{1}, \ldots, \sigma_{m}\right)$, is $C$ rich, with $C$ being a natural number, if it can be decomposed into at least $C$ consecutive, disjoint collision sequences in such a way that each of them is connected.

Proof of Theorem A. Let us fix a system satisfying the conditions of the Theorem where the number of particles is $N \geq 2$. The proof of the $K$ property of the standard billiard flow with $\mathcal{D}$-interaction will go on by induction on the number $N$ of particles. Assume thus that the standard billiard ball system with every permitted $\mathcal{D}$-interaction and in every permitted dimension has the $K$-property for any number of particles $n<N$ whenever the interior of the configuration space is connected. For $N=2$ this is known to be the case by $[\operatorname{Sin}(70)]$, [B-S $(73)](\nu=2)$ and by $[\mathrm{S}-\mathrm{Ch}(87)](\nu \geq 3)$.

It is important to note here that, in fact, the Bernoulli property of the standard billiard flow $\left(\mathbf{M},\left\{S^{t}\right\}, \mu\right)$ follows from its ergodicity, as it has been recently shown by Chernov-Haskell and Ornstein-Weiss, see [C-H (95)] and [O-W(95)]. For a brief summary of how the $K$-property follows, see, for instance, Section 7 of $[\operatorname{Sim}(92)-\mathrm{I}]$, or $[\mathrm{K}-\mathrm{S}(86)]$.

Richness will be understood in the sense of Definition 2.2 by choosing $C:=C(N)$ prescribed by the forthcoming Main Lemma 2.4. We stress 
again that the proof of Main Lemma 2.4 is independent of the inductive assumption.

The whole strategy outlined in Section 1 is based on the Theorem of Local Ergodicity for semi-dispersing billiards. In fact, the proof of global ergodicity uses its form given in [S-Ch (87)], whereas the inductive proof of part of the big proof requires its more general, transversal form as phrased in [K-S-Sz (90)] . For an easier identification of its conditions we refer to the work [K-S-Sz (90)] .

According to the results of $[\mathrm{V}(79)]$ and $[\mathrm{G}(81)]$, in a semi-dispersing billiard system there are no orbits with a finite accumulation point of collision moments. Consequently, Condition 2.1 of $[\mathrm{K}-\mathrm{S}-\mathrm{Sz}(90)]$ is fulfilled automatically. Condition 3.1, the so-called Chernov-Sinai Ansatz, will be verified at the end of the section. Condition 3.2 on the regularity of the set of degenerate tangencies is automatic for cylindric billiards. The argument given in [K-S-Sz (91)] for Condition 3.3 on the regularity of the set of double singularities for the case $N=3$ also works in our general case. What is more, Lemma 4.15 of [K-S-Sz (91)] asserts that the set of orbits containing two or more singular collisions is a countable union of codimension 2 submanifolds of $\mathbf{M}$. Thus, it is sufficient to concentrate our proof on the set $\mathbf{M}^{*}$ of orbits containing at most one singular collision and to show that, outside a slim set, any $x \in \mathbf{M}^{*}$ has an open neighbourhood belonging to one ergodic component. This is what we will do.

The fundamental theorem (Theorem on Local Ergodicity, Theorem 5 of [S-Ch (87)] or Corollary 3.12 of [K-S-Sz (90)] ) is applicable to our system of $N$ particles: a suitably small, open neighborhood of any sufficient phase point with at most one singularity belongs to one ergodic component.

Since the complement of a slim set in a connected manifold is always connected (see Property 2.10 from [K-S-Sz (91)] ), our goal is to show that, apart from a slim set of points, every phase point with at most one singularity is sufficient. To do so, let us follow our three step strategy outlined in the introduction. Let us recall first Theorem 5.1 of [Sim(92)-I], whose straightforward application to billiard ball systems with our restricted interactions is

Proposition 2.3. (Theorem 5.1 of $[\operatorname{Sim}(92)-\mathrm{I}]$ ) Consider a system of $N$ $(\geq 3)$ particles on the unit torus $\mathbb{T}^{\nu}(\nu \geq 2)$ satisfying the conditions of Theorem A. Assume that for all $n<N$ the statement of Theorem A holds true. Let $P$ be a given, 2-class partition of the $N$ particles. Then the set

$$
F_{+}=\left\{x \in \mathbf{M}: S^{[0, \infty)} x \text { is partitioned by } P\right\}
$$

is a closed, zero set with codimension at least two (i.e., a closed slim set). 
This statement would allow one to fix $C(N)$ (the number of connected graphs required for sufficiency) arbitrarily large.

The non-inductive, geometric-algebraic part of the strategy will be established by the following main lemma (the proof of which will be given in Section 3):

Main Lemma 2.4. Assume that the graph of interactions is a tree with edge degree $D$. If the symbolic collision sequence $\Sigma=\left(\sigma_{1}, \ldots, \sigma_{m}\right)\left(\sigma_{k} \in\right.$ $\mathcal{E}(\mathcal{G}))$ of the non-singular orbit segment $S^{[a, b]} x_{0}$ is 3-rich, then there exists an open neighbourhood $U\left(x_{0} \in U \subset M\right)$ and a closed algebraic subset $\mathcal{N} \subset U$ such that

(i) for every $y \in U \backslash \mathcal{N}$ the orbit segment $S^{[a, b]} y$ is sufficient;

(ii)

$$
\operatorname{codim}_{U} \mathcal{N} \geq \nu-D
$$

Consider now a rich point $x \in \mathbf{M}^{0}$. (By Proposition 2.3, we can assume the richness of $x$.) If $x \in \mathbf{M}^{0}$ is not sufficient, then by Main Lemma 2.4, there is an open ball-like neighbourhood $U$ of $x$ and a CW-complex (more accurately, a closed algebraic subset) $\mathcal{N} \subset U, x \in \mathcal{N}$, such that $\operatorname{codim}_{U} \mathcal{N} \geq$ 2 , and all non-sufficient points of $U$ are contained in $\mathcal{N}$. Consequently, by the Theorem on Local Ergodicity and by the connectedness of the set $U \backslash \mathcal{N}$, the neighborhood $U$ belongs to one ergodic component (modulo zero sets, of course).

Let us finally handle the phase points $x \in \mathbf{M}^{1}$. We can, and do, assume that $x$ belongs, for instance, to the set $\mathcal{S R}^{+}$. Here we recall Theorem 6.1 of $[\operatorname{Sim}(92)-\mathrm{I}]$, whose straightforward application to our situation is

Proposition 2.5. (Theorem 6.1 of [Sim(92)-I]) Suppose that for all $n<$ $N$ the statement of Theorem A holds true and, moreover, Main Lemma 2.4 has been proved for billiard flows satisfying the conditions of Theorem A. Then, for any given 2-class partition $P$ of the $N$ particles, any positive number $t_{0}$, and any cell $C \subset \mathcal{S} \mathcal{R}^{+}$of $\mathcal{S R}^{+}$with the maximum dimension $(2 d-3)$, the subset

$$
C\left(t_{0}, P\right)=\left\{x \in C: S^{\left[t_{0}, \infty\right)} x \text { is partitioned by } P\right\}
$$

has measure zero with respect to the Riemannian volume $\mu_{C}$ of the manifold $C$.

As said before, the set of doubly singular phase points is a countable union of codimension 2 submanifolds; hence, the condition of regularity of the orbit $S^{[0, \infty)} x$ can be omitted from the definition of $C\left(t_{0}, P\right)$. (It was 
present in the original publication.) Since the set $C\left(t_{0}, P\right)$ in this theorem is closed, it is a slim subset of $\mathbf{M}$; hence, we may assume that $x$ does not belong to any of these sets, i.e., the (non-singular) positive semi-trajectory $S^{(0, \infty)} x$ contains infinitely many consecutive, connected collision graphs. By Main Lemma 2.4, this positive orbit is sufficient, if only $x$ does not belong to some codimension 1 submanifolds of $\mathcal{S R}^{+}$, but such sets are slim (negligible) in the whole phase space $\mathbf{M}$. In this way we have proved two things:

(a) the Chernov-Sinai Ansatz, and the fact that

(b) apart from a slim subset of the phase space, the trajectory of every phase point $x \in \mathbf{M}^{1}$ is sufficient.

The inductive proof of the Theorem A is now complete.

Proof of Theorem B. The proof of Theorem B is completely analogous to that of Theorem A. Our first remark is that there the information on the graph of interactions was essentially used only in the proof of Main Lemma 2.4. Observe next that, if we want to obtain the statement of Theorem B for a given $N$, then the statements of Propositions 2.3 and 2.5 will still be true for our cyclic interaction. The reason is that, if a semi-trajectory of our system decomposes into two or more non-interacting subsystems, then the interaction graphs of these subsystems are simple trees (in fact, just paths). The only remaining task is to replace Main Lemma 2.4, and this is done by

Main Lemma 2.7. Assume that the graph of interactions consists of one circle of length $N$. If the symbolic collision sequence $\Sigma=\left(\sigma_{1}, \ldots, \sigma_{m}\right)$ $\left(\sigma_{k} \in \mathcal{E}(\mathcal{G})\right)$ of the non-singular orbit segment $S^{[a, b]} x_{0}$ is 4 -rich, then there exist an open neighbourhood $U\left(x_{0} \in U \subset M\right)$ and an algebraic set $\mathcal{N} \subset U$ such that

(i) for every $y \in U \backslash \mathcal{N}$ the orbit segment $S^{[a, b]} y$ is sufficient;

(ii)

$$
\operatorname{codim}_{U} \mathcal{N} \geq \nu-2
$$

Main Lemma 2.7 will be demonstrated separately in Section 4 . Hence Theorem B.

Proof of Theorem C. Our arguments will essentially follow the line of the proof of Theorem A, but an additional difficulty arises: since the statement is weaker so is the inductive hypothesis. As remarked earlier, the basic pillars of the induction are Proposition 2.3, Main Lemma 2.4 and Proposition 2.5. The codimension 1 statement of Main Lemma 2.4 is sufficient for 
our purposes in the neighbourhoods of rich points, and, consequently, we can concentrate on replacing Propositions 2.3 and 2.5.

Let us start with the first one.

Proposition 2.8. Consider a system of $N(\geq 3)$ particles on the unit torus $\mathbb{T}^{\nu}(\nu=D+1)$ satisfying the conditions of Theorem C. Assume that for all $n<N$ the statement of Theorem $C$ holds true. Let $P$ be a given, 2-class partition of the $N$ particles. Then the set

$$
F_{+}=\left\{x \in \mathbf{M}: S^{[0, \infty)} x \text { is partitioned by } P\right\}
$$

has measure zero.

Proof of Proposition 2.8. The proof borrows its approach from the proof of Theorem 5.1 of [Sim (92-I)], and therefore our exposition will be concise. Assume that the two classes are $P_{1}=\{1, \ldots, n\}$ and $P_{2}=\{n+1, \ldots, N\}$. The cases a) $\left|P_{1}\right|,\left|P_{2}\right| \geq 2$ and b) either $\left|P_{1}\right|$ or $\left|P_{2}\right|=1$ can be treated similarly and thus we only consider the first one.

It is, of course, sufficient to show that for an arbitrary small, open, balllike set $U_{0} \subset \mathbf{M}$ one has $\mu\left(U_{0} \cap F_{+}\right)=0$. Every point $x \in U_{0}$ can be characterized locally by the following coordinates in a unique way:

$$
\begin{array}{ll}
\text { (1) } \pi_{P_{1}}(x) \in \mathbf{M}_{1}, & \text { (2) } \pi_{P_{2}}(x) \in \mathbf{M}_{2}, \\
\text { (3) } C_{1}(x)=\frac{1}{n} \sum_{i=1}^{n} q_{i}(x) \in \mathbb{T}^{\nu}, & \text { (4) } I_{1}(x)=\frac{1}{n} \sum_{i=1}^{n} v_{i}(x), \\
\text { (5) } E_{1}(x)=\frac{1}{2} \sum_{i=1}^{n} v_{i}^{2}(x) . &
\end{array}
$$

Assume that, according to the above splitting of the data, $U_{0}$ is of the form

$$
U_{0}=B_{1} \times B_{2} \times B_{3} \times B_{4} \times B_{5},
$$

where $B_{1} \subset \mathbf{M}_{1}, B_{2} \subset \mathbf{M}_{2}, B_{3} \subset \mathbb{T}^{\nu}, B_{4} \subset \mathbb{R}^{\nu}, B_{5} \subset \mathbb{R}_{+}$are balls of the corresponding coordinate spaces. Choose and fix any $b_{i} \in B_{i}: i=3,4,5$. The relation $\mu\left(F_{+}\right)=0$ will certainly follow if we show that for almost every such choices of the $b_{i}$ 's

$$
\mu_{b_{3}, b_{4}, b_{5}}\left(F_{+}\left(b_{3}, b_{4}, b_{5}\right)\right)=0
$$

where

$$
F_{+}\left(b_{3}, b_{4}, b_{5}\right)=\left\{\left(y_{1}, y_{2}\right) \in B_{1} \times B_{2}:\left(y_{1}, y_{2}, b_{3}, b_{4}, b_{5}\right) \in F_{+}\right\}
$$


and $\mu_{b_{3}, b_{4}, b_{5}}$ denotes the conditional measure of $\mu$ under the conditions corresponding to fixing the values of $b_{3}, b_{4}, b_{5}$. (This conditional measure is equivalent to the Liouville measure on $\mathbf{M}_{1} \times \mathbf{M}_{2}$.) Indeed, we will show that (2.9) holds whenever the components of $b_{4}=I_{1}(x)$ are independent over the rationals.

The relation $x \in F_{+}$is equivalent to saying that for every pair $i \in P_{1}, j \in$ $P_{2},\{i, j\} \in \mathcal{E}$ and every $t \geq 0$,

$$
\varrho\left(q_{i}^{t}(x), q_{j}^{t}(x)\right) \geq d_{i j},
$$

where $\varrho(.,$.$) denotes the euclidean distance in the torus \mathbb{T}^{\nu}$.

Now we will be considering the subdynamics corresponding to our 2class partition (cf. the "Subsystems, decompositions" part of Section 2 in the paper [Sim(92)-I]), and will denote them, for simplicity, by $S_{1}$ and $S_{2}$, respectively (their phase spaces are $\mathbf{M}_{1}$ and $\mathbf{M}_{2}$, of course). Denote by $\widetilde{q}_{i}^{t}(x)$ the $i$ th configuration vector coordinate of $S_{1}^{t}\left(\pi_{P_{1}}(x)\right)$ if $i \in P_{1}$, and similarly by $\widetilde{q}_{j}^{t}(x)$ the $j$ th configuration vector coordinate of $S_{2}^{t}\left(\pi_{P_{2}}(x)\right)$ if $j \in P_{2}$. Since for $i \in P_{1}$,

$$
q_{i}^{t}(x)=\widetilde{q}_{i}^{t}(x)+C_{1}(x)+t I_{1}(x) \quad\left(\bmod \mathbb{Z}^{\nu}\right),
$$

and for $j \in P_{2}$,

$$
q_{j}^{t}(x)=\widetilde{q}_{j}^{t}(x)-\frac{n}{N-n} C_{1}(x)-\frac{n t}{N-n} I_{1}(x) \quad\left(\bmod \mathbb{Z}^{\nu}\right),
$$

(2.10) can be replaced by

$$
\varrho\left(\widetilde{q}_{i}^{t}(x)-\widetilde{q}_{j}^{t}(x),-\frac{N}{N-n} C_{1}(x)-\frac{N t}{N-n} I_{1}(x)\right) \geq d_{i j},
$$

where, needless to say, the second argument should be understood mod $\mathbb{Z}^{\nu}$. We also note that $C_{1}(x) /(N-n)$ is not defined uniquely, but our arguments are independent of the choice of its value.

Consider the product dynamics

$$
S^{t}\left(\pi_{P_{1}}(x)\right) \times S^{t}\left(\pi_{P_{2}}(x)\right),
$$

and choose an arbitrary pair $G_{1} \subset \mathbf{M}_{1}, G_{2} \subset \mathbf{M}_{2}$ of open subsets such that the factors of the previous product are $K$-flows on $G_{1}$ and $G_{2}$, respectively. As a consequence, the product is a mixing flow on the open subset $G_{1} \times G_{2} \subset$ $\mathbf{M}_{1} \times \mathbf{M}_{2}$. Let us denote by $\tilde{G}_{1}$ the projection of $G_{1}$ to the configuration vector coordinate $\tilde{q}_{i}$ living on $\mathbb{T}^{\nu}$ and similarly by $\tilde{G}_{2}$ the projection of $G_{2}$ 
into $\mathbb{T}^{\nu}$ corresponding to $\tilde{q}_{j}$. Of course, both $\tilde{G}_{1}$ and $\tilde{G}_{2}$ are non-empty, open subsets of $\mathbb{T}^{\nu}$, and so is $\tilde{G}_{1}-\tilde{G}_{2}$. Choose an open ball $G^{*} \subset \tilde{G}_{1}-\tilde{G}_{2}$ whose radius is less than $d_{i, j} / 2$. Having fixed the values of $b_{3} \in B_{3}, b_{4} \in$ $B_{4}, b_{5} \in B_{5}$ (with $b_{4}$ having rationally independent coordinates), we can find an infinite sequence $0<t_{1}<t_{2}<\cdots<t_{k}<\ldots, t_{k} \rightarrow \infty$, of time moments such that

$$
-\frac{N}{N-n} C_{1}(x)-\frac{N t_{k}}{N-n} I_{1}(x) \in G^{*} \quad\left(\bmod \quad \mathbb{Z}^{\nu}\right)
$$

for all $k=1,2, \ldots$ By the weak lemma on avoiding of balls (see Lemma 2.16 of $[\mathrm{K}-\mathrm{S}-\mathrm{Sz}(91)])$, the $\mu_{b_{3}, b_{4}, b_{5}}$-measure of those phase points of our previously defined product dynamics, for which $\tilde{q}_{i}-\tilde{q}_{j}$ never enters the set $G^{*}$ in the time moments $t_{k}: k=1,2, \ldots$, is zero. Hence $(2.9)$ and Proposition 2.8 follow.

Finally, we formulate a proposition to replace Proposition 2.5.

Proposition 2.11. Consider a system of $N(\geq 3)$ particles on the unit torus $\mathbb{T}^{\nu} \quad(\nu=D+1)$ satisfying the conditions of Theorem $C$. Assume, moreover, that for every $n<N$ the statement of Theorem $C$ holds true. Then for any given 2-class partition $P$ of the $N$ particles, any positive number $t_{0}$ and any cell $C \subset S \mathcal{R}^{+}$of $S \mathcal{R}^{+}$with the maximum dimension $(2 d-3)$ the subset $C\left(t_{0}, P\right)$ defined by (2.6) has measure zero with respect to the Riemannian volume $\mu_{c}$ of the manifold $C$.

Proof of Proposition 2.11. The proof given for Theorem 6.1 of Sim (92-I) also works under our conditions. The basic argument is that the inductive assumption ensures that the factors corresponding to the subdynamics defined by the 2-class partition have positive ergodic components, and the whole proof can be restricted to products of these positive components. On these product sets the basic hyperbolic structure, fundamental for the proof, is the same as in the case of the product of $K$-flows.

Thus, we get Proposition 2.11

Consequently, Theorem $\mathrm{C}$ is established.

Finally, the proof of Theorem D can be obtained from that of Theorem C just like Theorem B was established after the proof of Theorem A.

\section{Combinatorial richness implies sufficiency.}

The content of this paragraph is the proof of Main Lemma 2.4 which heavily uses the upcoming Lemma 3.2. 
We denote by

$$
A_{k}: \mathcal{N}_{a}\left(S^{[a, b]} x_{0}\right) \rightarrow \mathbb{R}
$$

the advance (time shift) corresponding to the $k$ th collision $\sigma_{k}$ (see Section 2 of $[\operatorname{Sim}(92)-\mathrm{I}]$, especially formulae $(2.16)-(2.17)$ ). It is known (see, for instance, Section 2 of $[\operatorname{Sim}(92)-\mathrm{I}])$ that $A_{k}$ is a linear functional on the real vector space $\mathcal{N}_{a}\left(S^{[a, b]} x_{0}\right)$.

Lemma 3.2. If $1 \leq p<q<r \leq m, \sigma_{p}=\sigma_{r}, \sigma_{p} \neq \sigma_{q}$, and $\sigma_{p} \cap \sigma_{q} \neq \emptyset$, then $A_{p}=A_{q}=A_{r}$ modulo a closed algebraic set of exceptional phase points $x_{0}$, such that the codimension (in $\mathbf{M}$, as always!) of this exceptional set is at least $\nu-D$.

Proof. Induction on the number $r-p$. For $r-p=1$ the statement is vacuous. (Or, in other words, if we considered $r-p=2$ as the starting point of the induction, its proof would be exactly the same as the inductive step.) Let now $r-p>1$, and suppose the inductive hypothesis for smaller values of $r-p$.

Plainly, we can assume that $\sigma_{k} \neq \sigma_{p}$ for $k=p+1, \ldots, r-1$. Write down the Connecting Path Formula (CPF, Lemma 2.9 of [Sim(92)-II]) for the new advance $A_{r}$ in terms of $A_{p}$ and the advances $A_{k}$ with $p<k<r$ and $\sigma_{k} \cap \sigma_{p} \neq \emptyset$ :

$$
\begin{gathered}
A_{r}\left(v_{i_{p}}^{-}\left(t_{r}\right)-v_{j_{p}}^{-}\left(t_{r}\right)\right)=A_{p}\left(v_{i_{p}}^{+}\left(t_{p}\right)-v_{j_{p}}^{+}\left(t_{p}\right)\right)+ \\
\sum_{\substack{p<k<r \\
i_{p} \in \sigma_{k}}} A_{k}\left(v_{i_{p}}^{+}\left(t_{k}\right)-v_{i_{p}}^{-}\left(t_{k}\right)\right)-\sum_{\substack{p<k<r \\
j_{p} \in \sigma_{k}}} A_{k}\left(v_{j_{p}}^{+}\left(t_{k}\right)-v_{j_{p}}^{-}\left(t_{k}\right)\right) .
\end{gathered}
$$

According to our inductive hypothesis, the advances $A_{k}$ in (3.3) only depend on the edge $\sigma_{k}$, and they are independent of the actual value of $k$. Thus, the terms in (3.3) with the same $\sigma_{k}$ can be collected together, so we obtain

$$
\begin{aligned}
& \text { (3.4) } A_{r}\left(v_{i_{p}}^{-}\left(t_{r}\right)-v_{j_{p}}^{-}\left(t_{r}\right)\right)=A_{p}\left(v_{i_{p}}^{+}\left(t_{p}\right)-v_{j_{p}}^{+}\left(t_{p}\right)\right)+ \\
& \sum_{\substack{\sigma \in \mathcal{E} \\
i_{p} \in \sigma}} A_{\sigma} \cdot \sum_{\substack{p<k<r \\
\sigma_{k}=\sigma}}\left(v_{i_{p}}^{+}\left(t_{k}\right)-v_{i_{p}}^{-}\left(t_{k}\right)\right)-\sum_{\substack{\sigma \in \mathcal{E} \\
j_{p} \in \sigma}} A_{\sigma} \cdot \sum_{\substack{p<k<r \\
\sigma_{k}=\sigma}}\left(v_{j_{p}}^{+}\left(t_{k}\right)-v_{j_{p}}^{-}\left(t_{k}\right)\right) .
\end{aligned}
$$

Suppose now that, to the contrary of the lemma, $A_{p} \neq A_{r}$. Evaluate (3.4) at a neutral vector $n_{0} \in \mathcal{N}_{a}\left(S^{[a, b]} x_{0}\right)$ for which, say, $A_{p}\left(n_{0}\right)=0$ 
but $A_{r}\left(n_{0}\right) \neq 0$. (This can always be achieved by adding to $n_{0}$ an appropriate multiple of the flow direction.) Formula (3.4) evaluated at such $n_{0} \in \mathcal{N}_{a}\left(S^{[a, b]} x_{0}\right)$ yields

$$
A_{r}\left(n_{0}\right)\left(v_{i_{p}}^{-}\left(t_{r}\right)-v_{j_{p}}^{-}\left(t_{r}\right)\right)=\sum_{\substack{\sigma \in \mathcal{E} \\ i_{p} \in \sigma}} A_{\sigma}\left(n_{0}\right) \Gamma_{\sigma}-\sum_{\substack{\sigma \in \mathcal{E} \\ j_{p} \in \sigma}} A_{\sigma}\left(n_{0}\right) \Gamma_{\sigma}
$$

where the vector $\Gamma_{\sigma}$ is defined by the formula

$$
\Gamma_{\sigma}=\sum_{\substack{p<k<r \\ \sigma k=\sigma \\ \sigma \cap \sigma_{p}=\alpha}}\left(v_{\alpha}^{+}\left(t_{k}\right)-v_{\alpha}^{-}\left(t_{k}\right)\right)
$$

Of course, in the equations (3.4-3.5) we only include the terms $A_{\sigma} \cdot \Gamma_{\sigma}$ with collisions $\sigma$ for which the sum in (3.6) is non-empty. Correspondingly, in the remaining part of the proof of Lemma 3.2, when speaking about $A_{\sigma}$ or $\Gamma_{\sigma}$, we always mean by $\sigma$ a collision with a non-empty sum (3.6).

By our assumption on the graph $\mathcal{G}$, the number of terms on the righthand side does not exceed $D$. We observe that the vectors $\Gamma_{\sigma}$ from (3.6) are determined the evolution of two disjoint systems evolving between the collisions $\sigma_{p}$ and $\sigma_{r}$, and these two systems get connected by the collision $\sigma_{r}=\left\{i_{r}, j_{r}\right\}=\left\{i_{p}, j_{p}\right\}$. Thus, the relative velocity $\left(v_{i_{p}}^{-}\left(t_{r}\right)-v_{j_{p}}^{-}\left(t_{r}\right)\right)$ on the left-hand side of (3.5) can be chosen independently of the vectors $\Gamma_{\sigma}$. Therefore, the event that $\left(v_{i_{p}}^{-}\left(t_{r}\right)-v_{j_{p}}^{-}\left(t_{r}\right)\right)$ belongs to the linear subspace of $\mathbb{R}^{\nu}$ generated by the vectors $\Gamma_{\sigma}$ (which is the assertion of (3.5)) can only occur on a codimension $(\nu-D)$ subset of the phase space.

Hence $A_{p}=A_{r}$ modulo a codimension $(\nu-D)$ closed algebraic set.

Let us turn now to the proof of $A_{p}=A_{q}$. It requires a bit more detailed analysis of the vectors $\Gamma_{\sigma}$ on the right-hand side of (3.4). Namely, the inductive assumption not only guarantees that the advance $A_{k}$ of $\sigma_{k}$ merely depends on the unordered pair $\sigma_{k}=\sigma$, but, also, it says that $A_{\sigma^{*}}=A_{\sigma^{* *}}$ whenever there exist integers $k_{1}^{*}, k_{2}^{*}, k^{* *}$ among the integers $p+1, \ldots, r-1$, and there exists an element $\alpha \in\left\{i_{p}, j_{p}\right\}$ such that $\alpha \in \sigma^{*} \cap \sigma^{* *}, k_{1}^{*}<k^{* *}<$ $k_{2}^{*}, \sigma^{*}=\sigma_{k_{1}^{*}}=\sigma_{k_{2}^{*}}$, and $\sigma^{* *}=\sigma_{k^{* *}}$. On the set of edges (collisions)

$$
E=\left\{\sigma \in \mathcal{E} \mid \sigma \cap\left\{i_{p}, j_{p}\right\} \neq \emptyset \text { and } \exists k, p<k<r, \sigma=\sigma_{k}\right\}
$$

we define the equivalence relation $\sim$ as the smallest equivalence relation on $E$ making every pair of $\sigma^{*}$ and $\sigma^{* *}$ above equivalent. Let $E=C_{1} \cup$ $\cdots \cup C_{s}$ be the splitting of $E$ into the equivalence classes of $\sim$. Thanks to 
the inductive hypothesis, we can pull together the terms $A_{\sigma} \cdot \Gamma_{\sigma}$ of (3.4) corresponding to the same class $C_{i}$ of $\sim$ as follows:

$$
A_{r}\left(v_{i_{p}}^{-}\left(t_{r}\right)-v_{j_{p}}^{-}\left(t_{r}\right)\right)=A_{p}\left(v_{i_{p}}^{+}\left(t_{p}\right)-v_{j_{p}}^{+}\left(t_{p}\right)\right)+\sum_{i=1}^{s} A\left(C_{i}\right) \cdot \Gamma\left(C_{i}\right)
$$

where $A\left(C_{i}\right)=A_{\sigma}$ for any $\sigma \in C_{i}$, and $\Gamma\left(C_{i}\right)=\sum_{\sigma \in C_{i}} \Gamma_{\sigma}$ if $i_{p} \in \sigma$ for the elements $\sigma$ of $C_{i}$, while $\Gamma\left(C_{i}\right)=-\sum_{\sigma \in C_{i}} \Gamma_{\sigma}$ otherwise. The next sublemma shows that the advantage of the expansion (3.8) is that the terms $\Gamma\left(C_{i}\right) \in \mathbb{R}^{\nu}$ become independent of each other.

Sublemma 3.9. Let $C_{i}$ and $C_{j}(1 \leq i<j \leq s)$ be two $\sim$ equivalence classes with the property

$$
\bigcap_{\sigma \in C_{i} \cup C_{j}} \sigma \neq \emptyset
$$

i.e., the edges of $C_{i}$ and $C_{j}$ are all adjacent to $i_{p}$ or they are all adjacent to $j_{p}$. Then either for every pair $k, l \in\{p+1, \ldots, r-1\}$ the relations $\sigma_{k} \in C_{i}$ and $\sigma_{l} \in C_{j}$ imply $k<l$, or for every pair $k, l \in\{p+1, \ldots, r-1\}$ the relations $\sigma_{k} \in C_{i}$ and $\sigma_{l} \in C_{j}$ imply $k>l$, that is, there is a natural time ordering among the classes $C_{i}$ containing the edges adjacent to $i_{p}\left(j_{p}\right)$.

The proof is a straightforward consequence of the definition of the equivalence relation $\sim$.

We can now finish the proof of Lemma 3.2. Suppose that, contrary to the statement of the lemma, $A_{q}\left(n_{0}\right) \neq 0, A_{p}\left(n_{0}\right)=A_{r}\left(n_{0}\right)=0$ with some vector $n_{0} \in \mathcal{N}_{a}\left(S^{[a, b]} x_{0}\right)$. Evaluate (3.8) at this vector $n_{0}$ :

$$
\sum_{i=1}^{s} A\left(C_{i}\right)\left[n_{0}\right] \cdot \Gamma\left(C_{i}\right)=0 .
$$

Since $A\left(C_{i}\right)\left[n_{0}\right] \neq 0$ for $\sigma_{q} \in C_{i}$, the equation (3.10) means that the vectors

$$
\Gamma\left(C_{1}\right), \ldots, \Gamma\left(C_{s}\right)\left(\in \mathbb{R}^{\nu}\right)
$$

are linearly dependent. According to the previous sublemma and the fact that the graph $\mathcal{G}$ is a tree, the velocity changes $\Gamma\left(C_{1}\right), \ldots, \Gamma\left(C_{s}\right)$ may be chosen independently. Taking into account the inequality $s \leq D$, we have that the linear dependence (3.10) can only occur on a set of phase points $x_{0}$ with codimension at least $\nu+1-D$. Hence Lemma 3.2.

Finishing the proof of Main Lemma 2.4. Let $\Sigma^{(1)}=\left(\sigma_{1}, \ldots, \sigma_{k}\right), \Sigma^{(2)}=$ $\left(\sigma_{k+1}, \ldots, \sigma_{l}\right)$, and $\Sigma^{(3)}=\left(\sigma_{l+1}, \ldots, \sigma_{m}\right)$, be subgraphs of $\Sigma$ all connected 
on the vertex set $\mathcal{V}=\{1,2, \ldots, N\}$, i.e., the set of edges of $\Sigma^{(j)}(j=1,2,3)$ is the set $\mathcal{E}$ of all edges of $\mathcal{G}$. The fact that $\mathcal{G}$ is a tree and Lemma 3.2 straightforwardly imply that all advances $A_{1}, \ldots, A_{m}$ must be equal modulo a closed algebraic set of exceptional phase points $x_{0}$ with codimension at least $\nu-D$. The identity of these advances, however, precisely means that the orbit segment $S^{[a, b]} x_{0}$ is sufficient, see also Lemma 2.13 of [Sim(92)-II]. This finishes the proof of Main Lemma 2.4.

\section{Trajectories with a circular collision graph.}

In this section we slightly generalize the results of the previous paragraph by considering the following graph $\mathcal{G}_{c}=\left(\mathcal{V}, \mathcal{E}_{c}\right)$ of the circular interaction:

$$
\mathcal{E}_{c}=\{\{i, i+1\} \mid 1 \leq i \leq N-1\} \cup\{\{1, N\}\}
$$

For this graph $\mathcal{G}_{c}$, which is a circle of length $N$, the edge degree $D$ is equal to two. The actual importance of this circular interaction is explained by the fact that this is just the interaction of the particles in the so-called pencase model introduced by Chernov and Sinai, see [S-Ch(85)] or Section 1.

The content of this section is exclusively the proof of Main Lemma 2.7.

Before proving anything, however, it is worth noting that the whole issue of proving sufficiency by the use of combinatorial richness is totally insensitive of the uniform velocity dilations of the billiard flow. Therefore, it is not necessary to assume the usual energy normalization $\sum_{i=1}^{N} v_{i}^{2}=1$, so we will not do that.

First of all, we need the following

Lemma 4.2. Suppose that the graph of allowed collisions is the circle $\mathcal{G}_{c}$ and $S^{[a, b]} x_{0}$ is a non-singular orbit segment with the symbolic sequence $\left(\sigma_{1}, \ldots, \sigma_{m}\right)$. If $1 \leq p<q<r \leq m, \sigma_{p}=\sigma_{r}, \sigma_{p} \neq \sigma_{q}$, and $\sigma_{p} \cap \sigma_{q} \neq \emptyset$, then $A_{p}=A_{q}=A_{r}$ modulo a closed algebraic set of exceptional phase points $x_{0}$ such that the codimension of this set is at least $\nu-2$.

Proof of Lemma 4.2. This is again an induction on the number $r-p$. For $r-p=1$ there is nothing to prove. Suppose that $r-p>1$ and the statement is true for smaller values of $r-p$. Just like in the previous section, we can assume that $\sigma_{k} \neq \sigma_{p}$ for $k=p+1, \ldots, r-1$, and $\sigma_{p}=\{1, N\}$, i.e., $i_{p}=1$, $j_{p}=N$. The useful, short form of the Connecting Path Formula for the 
new advance $A_{r}$ in terms of the earlier advances is now

$$
\begin{gathered}
A_{r} \cdot\left(v_{1}^{-}\left(t_{r}\right)-v_{N}^{-}\left(t_{r}\right)\right)=A_{p} \cdot\left(v_{1}^{+}\left(t_{p}\right)-v_{N}^{+}\left(t_{p}\right)\right) \\
+A_{\{1,2\}} \cdot \sum_{\substack{k \\
p<k<r \\
\sigma_{k}=\{1,2\}}}\left(v_{1}^{+}\left(t_{k}\right)-v_{1}^{-}\left(t_{k}\right)\right) \\
-A_{\{N-1, N\}} \cdot \sum_{\substack{k \\
p<k<r \\
\sigma_{k}=\{N-1, N\}}}\left(v_{N}^{+}\left(t_{k}\right)-v_{N}^{-}\left(t_{k}\right)\right) \\
=A_{p} \cdot\left(v_{1}^{+}\left(t_{p}\right)-v_{N}^{+}\left(t_{p}\right)\right)+A_{\{1,2\}} \cdot\left(v_{1}^{-}\left(t_{r}\right)-v_{1}^{+}\left(t_{p}\right)\right) \\
-A_{\{N-1, N\}} \cdot\left(v_{N}^{-}\left(t_{r}\right)-v_{N}^{+}\left(t_{p}\right)\right) .
\end{gathered}
$$

Here again we used, as a corollary of the inductive hypothesis, the fact that the advance $A_{k}$ of a collision $\sigma_{k}(p<k<r)$ merely depends on $\sigma_{k}$ and not on the actual value of $k$.

First we rewrite the equation (4.3) with $n \in \mathcal{N}_{a}\left(S^{[a, b]} x_{0}\right)$, neutral vectors for which $A_{r}(n)=0$. Recall that any neutral vector can be modified by a scalar multiple of the flow direction in such a way that one achieves $A_{r}(n)=0$. Taking also into account that the multiplier of $A_{\{1,2\}}$ in (4.3) is the total change of velocity of the first ball between $\sigma_{p}$ and $\sigma_{r}$, and that a similar statement is true for the multiplier of $A_{\{N-1, N\}}$, we obtain

$$
\begin{aligned}
A_{p}(n)\left(v_{1}^{+}\left(t_{p}\right)-v_{N}^{+}\left(t_{p}\right)\right)+ & A_{\{1,2\}}(n)\left(v_{1}^{-}\left(t_{r}\right)-v_{1}^{+}\left(t_{p}\right)\right) \\
& -A_{\{N-1, N\}}(n)\left(v_{N}^{-}\left(t_{r}\right)-v_{N}^{+}\left(t_{p}\right)\right)=0,
\end{aligned}
$$

for $n \in \mathcal{N}_{a}\left(S^{[a, b]} x_{0}\right), A_{r}(n)=0$.

In order to finish the inductive proof of Lemma 4.2, it is just enough to prove that all three advances in (4.4) must be zero apart from a set of codimension at least $\nu-2$. This is what we do.

We may assume that every edge of the path $\mathcal{E}_{c} \backslash\{\{1, N\}\}$ occurs among the edges $\sigma_{p+1}, \ldots, \sigma_{r-1}$, otherwise the statement of Lemma 4.2 directly follows from Lemma 3.2.

In order to show that $A_{p}=A_{\{1,2\}}=A_{\{N-1, N\}}$ modulo a codimension $(\nu-2)$ set, use either the inductive hypothesis of Lemma 4.2, or just the assertion of Lemma 3.2 to construct an equivalence relation $\sim$ on the set of integers $\{p+1, \ldots, r-1\}$ as follows: $i \sim j(p+1 \leq i<j \leq r-1)$ if and only if $A_{i}=A_{j}$ logically follows from the statement of Lemma 3.2. The first observation is that the validity of $i \sim j$ merely depends on the collisions $\sigma_{i}$ and $\sigma_{j}$, and it is independent of the actual value of $i$ and $j$. This means 
that, in fact, the equivalence relation $\sim$ descends to an equivalence relation (also denoted by $\sim$ ) on the set of edges $\left\{\sigma_{p+1}, \ldots, \sigma_{r-1}\right\}=\mathcal{E}_{c} \backslash\{\{1, N\}\}$. Two adjacent edges $\{i-1, i\}$ and $\{i, i+1\}$ are $\sim$ equivalent $(2 \leq i \leq N-1)$ if and only if there are three integers $p+1 \leq k_{1}<k_{2}<k_{3} \leq r-1$ such that either $\sigma_{k_{1}}=\sigma_{k_{3}}=\{i-1, i\}, \sigma_{k_{2}}=\{i, i+1\}$, or $\sigma_{k_{1}}=\sigma_{k_{3}}=\{i, i+1\}$, $\sigma_{k_{2}}=\{i-1, i\}$. The equivalence relation $\sim$ is then the transitive hull of the above defined $\sim$ between adjacent edges.

A ball (vertex) $i \in\{2, \ldots, N-1\}$ is called a separating one if $\{i-1, i\} \not$ $\{i, i+1\}$. Let

$$
2 \leq i_{1}<i_{2}<\cdots<i_{s} \leq N-1
$$

be the collection of all separating vertices. It follows from the equation

$$
A_{\left\{i_{k-1}, i_{k-1}+1\right\}}=A_{\left\{i_{k-1}+1, i_{k-1}+2\right\}}=\cdots=A_{\left\{i_{k}-1, i_{k}\right\}}
$$

$\left(1 \leq k \leq s+1, i_{0}=1, i_{s+1}=N\right)$ that the subsystem of balls $\left\{i_{k-1}, \ldots, i_{k}\right\}$ has a sufficient trajectory segment on the closed time interval $\left[t^{\prime}, t^{\prime \prime}\right]$, where $t^{\prime}$ is the time of the first occurrence of any collision $\sigma_{n}(p+1 \leq n \leq r-1)$ connecting two vertices of the subsystem $\left\{i_{k-1}, \ldots, i_{k}\right\}$, while $t^{\prime \prime}$ is the time of the last such occurrence.

The assertion of Lemma 4.2 is obviously true for $s=0$. Thus, we assume that $s \geq 1$.

Consider the first separating vertex $i_{1}\left(2 \leq i_{1} \leq N-1\right)$. In order to prove the lemma, it is enough to show that $A_{\{1,2\}}=0$ in (4.4) modulo a codimension $(\nu-2)$ set, because the proof of $A_{\{N-1, N\}}=0$ is analogous, and the coefficient $A_{p}(n)$ cannot be the sole nonzero coefficient in (4.4).

We assume that every collision of type $\left\{i_{1}-1, i_{1}\right\}$ (in the time interval $\left(t_{p}, t_{r}\right)$, as always!) precedes every collision of type $\left\{i_{1}, i_{1}+1\right\}$ of the time interval $\left(t_{p}, t_{r}\right)$. (The handling of the opposite case is analogous.) Choose, therefore, a separating time $t^{*}$ for which every occurrence of the collision $\left\{i_{1}-1, i_{1}\right\}$ takes place before $t^{*}$ and, correspondingly, every occurrence of the collision $\left\{i_{1}, i_{1}+1\right\}$ takes place after $t^{*}$. For a while we assume that $i_{1} \geq 3$. After the proof of the equation $A_{\{1,2\}}=0$ in this case, we shall handle the case $i_{1}=2$ separately.

Execute a small phase perturbation (i.e., a configuration and velocity perturbation) at time $t^{*}$ such that the relative positions $q_{k}-q_{l}$ and the relative velocities $v_{k}-v_{l}$ are unchanged for $i_{1} \leq k<l \leq N$, otherwise these small perturbations may be arbitrary. Distinguish such a varying, perturbed system from the unperturbed one by the superscript over the kinetic data. Then a linear dependence (4.4) with nonzero coefficient $A_{\{1,2\}}(n)$ can be rewritten for the perturbed system as

$$
\begin{aligned}
& \tilde{v}_{1}^{-}\left(\tilde{t}_{r}\right)-\tilde{v}_{1}^{+}\left(\tilde{t}_{p}\right) \\
= & \alpha\left(\tilde{v}_{1}^{+}\left(\tilde{t}_{p}\right)-\tilde{v}_{i_{1}}\left(t^{*}\right)\right)+\alpha\left(\tilde{v}_{i_{1}}\left(t^{*}\right)-\tilde{v}_{N}^{+}\left(\tilde{t}_{p}\right)\right)+\beta\left(\tilde{v}_{N}^{-}\left(\tilde{t}_{r}\right)-\tilde{v}_{N}^{+}\left(\tilde{t}_{p}\right)\right)
\end{aligned}
$$


with some $\alpha, \beta \in \mathbb{R}$. The advantage of the form (4.5) is that the second and third terms on the right-hand side of it do not change under the considered perturbations, so we can omit the superscript ${ }^{\sim}$ from them:

$$
\begin{aligned}
& \tilde{v}_{1}^{-}\left(\tilde{t}_{r}\right)-\tilde{v}_{1}^{+}\left(\tilde{t}_{p}\right) \\
= & \alpha\left(\tilde{v}_{1}^{+}\left(\tilde{t}_{p}\right)-\tilde{v}_{i_{1}}\left(t^{*}\right)\right)+\alpha\left(v_{i_{1}}\left(t^{*}\right)-v_{N}^{+}\left(t_{p}\right)\right)+\beta\left(v_{N}^{-}\left(t_{r}\right)-v_{N}^{+}\left(t_{p}\right)\right)
\end{aligned}
$$

for some $\alpha, \beta \in \mathbb{R}$.

Denote by $\bar{S}^{\left[t_{p}, t_{r}\right]} y_{0}$ the time evolution of the varying, perturbed billiard subsystem $\left\{1, \ldots, i_{1}\right\}$ during the closed time interval $\left[t_{p}, t_{r}\right]$, when all (in principle) possible collisions of type $\left\{i_{1}, i_{1}+1\right\}$ or $\{1, N\}$ are cancelled. Also, this subsystem is observed, by definition, from a moving reference coordinate system attached to the center of mass of the particles $1,2, \ldots, i_{1}$. (The center of mass can be defined locally in a continuous manner.) Denote the kinetic data of this subsystem (observed from the abovementioned reference system) by $\bar{q}_{j}(t)$ and $\bar{v}_{j}(t), j=1, \ldots, i_{1}$. The linear dependence (4.6) can then be rewritten as

$$
\begin{aligned}
& \bar{v}_{1}^{-}\left(t_{r}\right)-\bar{v}_{1}^{+}\left(t_{p}\right) \\
= & \alpha\left(\bar{v}_{1}^{+}\left(t_{p}\right)-\bar{v}_{i_{1}}^{-}\left(t_{r}\right)\right)+\alpha\left(v_{i_{1}}\left(t^{*}\right)-v_{N}^{+}\left(t_{p}\right)\right)+\beta\left(v_{N}^{-}\left(t_{r}\right)-v_{N}^{+}\left(t_{p}\right)\right)
\end{aligned}
$$

with some $\alpha, \beta \in \mathbb{R}$.

We remind the reader here that

(i) the subsystem $\bar{S}^{\left[t_{p}, t_{r}\right]} y_{0}$ is sufficient, and

(ii) the perturbations $\sim$ defined at time $t^{*}$ make it possible to vary the subsystem's phase point $\bar{S}^{t^{*}} y_{0}$ on an open subset of the phase space of that subsystem.

The next sublemma is very easy to verify, and its proof will be left to the reader.

Sublemma 4.8. Let $T^{(c, d)} z_{0}$ be a non-singular and sufficient orbit segment of a standard billiard flow of $n$ balls, where $n \geq 3$. Let us execute all possible (but small!) pure configuration perturbations of this system at time $c$ that keep the velocity of the $k$ th ball $(k \neq 1)$ at time $d$ fixed. Then the velocity $v_{1}(d)$ of the first ball fills up an open subset of $\mathbb{R}^{\nu}$ with maximum rank $\nu$ if $n \geq 4$, and $v_{1}(d)$ fills up an open subset of a $(\nu-1)$-dimensional sphere with maximum rank $\nu-1$ if $n=3$.

The properties (i)-(ii) above and Sublemma 4.8 (applied to the subsystem of balls $\left.1, \ldots, i_{1}\right)$ immediately ensure that a linear dependence of sort 
(4.7) can only hold on a set of codimension at least $\nu-2$. The proof of the equation $A_{\{1,2\}}(n)=0$ (modulo a codimension $(\nu-2)$ set) is now proved in the case of $i_{1} \geq 3$.

Let us now take up the similar problem in the case of $i_{1}=2$. Then one cannot use Sublemma 4.8, so it must be replaced by the following

Sublemma 4.9. Let $T^{(c, d)} z_{0}$ be a non-singular and sufficient (that is, containing at least one collision) orbit segment of a standard billiard flow of 2 balls, where, as said before, we do not use the energy normalization. Then the only constraint interrelating the relative velocities $v_{1}(d)-v_{1}(c)$ and $v_{1}(c)-v_{2}(d)=v_{1}(c)+v_{1}(d)$ is that these two vectors must be orthogonal to each other.

The proof is by simple inspection.

The consequence of this sublemma is that in the case of $i_{1}=2$ either both vectors $v_{1}^{+}\left(t_{p}\right)-v_{N}^{+}\left(t_{p}\right)$ and $v_{N}^{-}\left(t_{r}\right)-v_{N}^{+}\left(t_{p}\right)$ are perpendicular to the vector $v_{1}^{+}\left(t_{p}\right)-v_{2}\left(t^{*}\right)$ or, if not so, then the validity of a linear dependence (4.7) implies that the relative velocity on the left-hand side of (4.7) must belong to a line if the relative velocities on the right-hand side of (4.7) are fixed. As a direct inspection shows, however, either possibility can only occur on a codimension $(\nu-2)$ subset of the phase space. This shows that $A_{\{1,2\}}(n)=0$ (modulo a codimension $(\nu-2)$ set) in the case of $i_{1}=2$.

The proof of Lemma 4.2 is now finished.

Finishing the proof of Main Lemma 2.7.

Let the symbolic collision sequence $\Sigma=\left(\Sigma_{1}, \ldots, \Sigma_{4}\right)$ be composed of the four consecutive, connected subsequences $\Sigma_{j}, j=1,2,3,4$, and study the non-singular orbit segment $S^{[a, b]} x_{0}$ of the billiard flow with the restricted $\mathcal{G}_{c}$-interaction such that the symbolic collision sequence of $S^{[a, b]} x_{0}$ is $\Sigma$.

Due to the connectedness, the subsequence $\Sigma_{2}$ contains the edges of a full path (of length $N-1$ ) on the vertex set $\{1,2, \ldots, N\}$. By relabelling the particles we may assume that $\{i, i+1\} \in \Sigma_{2}$ for $i=1, \ldots, N-1$.

Consider an integer $k, 2 \leq k \leq N-1$, and show that $A_{\{k-1, k\}}=$ $A_{\{k, k+1\}}$. If either of the edges $\{k-1, k\},\{k, k+1\}$ is in the intersection $\Sigma_{1} \cap \Sigma_{3}$, then $A_{\{k-1, k\}}=A_{\{k, k+1\}}$ by Lemma 4.2. Thus we can assume that $\{k-1, k\} \notin \Sigma_{1} \cap \Sigma_{3}$ and $\{k, k+1\} \notin \Sigma_{1} \cap \Sigma_{3}$. Thanks to the connectedness of $\Sigma_{1}$ and $\Sigma_{3}$, any of these two graphs contains one of the edges $\{k-1, k\}$ and $\{k, k+1\}$. Therefore, we may assume that $\{k-1, k\} \notin$ $\Sigma_{3}$ and $\{k, k+1\} \notin \Sigma_{1}$. By analogous reasoning $\{k-1, k\} \notin \Sigma_{4}$.

Consider now the common edge set $\mathcal{E}_{c} \backslash\{\{k-1, k\}\}$ of $\Sigma_{3}$ and $\Sigma_{4}$. Since $\Sigma_{1} \cup \Sigma_{2}$ contains the entire edge set $\mathcal{E}_{c}$ of $\mathcal{G}_{c}$, again by Lemma 4.2 we have that the advances of any two adjacent edges of $\Sigma_{3}$ are equal. However, this implies the sufficiency, in particular $A_{\{k-1, k\}}=A_{\{k, k+1\}}$, just what we 
wanted to show. Since these arguments break down only at phase points outside a codimension $(\nu-2)$ subset of the phase space, the proof of Main Lemma 2.7 is now complete.

\section{References}

[B-L-P-S(92)] L. Bunimovich, C. Liverani, A. Pellegrinotti and Yu. Sukhov, Special systems of hard balls that are ergodic, Commun. Math. Phys. 146, 357396.

[B-S(73)] L. A. Bunimovich and Ya. G. Sinai, The fundamental theorem of the theory of scattering billiards, Math. USSR-Sb. 19, 407-423.

[C-H(95)] N. I. Chernov and C. Haskell, Nonuniformly hyperbolic K-systems are Bernoulli, to appear in Ergodic Theory and Dynamical Systems.

[G(81)] G. Galperin, On systems of locally interacting and repelling particles moving in space, Trudy MMO 43, 142-196.

[H-W(48)] W. Hurewicz and H. Wallman, Princeton Mathematical Series 4.

[K-S(86)] A. Katok and J. M. Strelcyn, Invariant manifolds, entropy and billiards; smooth maps with singularities, Lecture Notes in Mathematics 1222, Springer, 1986.

[K-S-Sz(89)] A. Krámli, N. Simányi and D. Szász, Ergodic properties of semi-dispersing billiards I, two cylindric scatterers in the 3-d torus, Nonlinearity 2, 311326.

[K-S-Sz(90)] _ A "transversal" fundamental theorem for semi-dispersing billiards, Commun. Math. Phys. 129, 535-560.

[K-S-Sz(91)] 133, 37-72.

[K-S-Sz(92)] , The K-property of four billiard balls, Commun. Math. Phys. 144, 107-148.

[O-W(95)] D. Ornstein and B. Weiss, On the Bernoulli nature of systems with some hyperbolic structure, manuscript, p. 23.

[Sim(92)] N. Simányi, The K-property of $N$ billiard balls I, Invent. Math. 108, 521-548; II., ibidem 110, 151-172.

[Sin(63)] Ya. G. Sinai, On the foundation of the ergodic hypothesis for a dynamical system of statistical mechanics, Soviet Math. Dokl. 4, 1818-1822.

[Sin(70)] _ Dynamical systems with elastic reflections, Russian Math. Surveys 25:2, 137-189.

[Sin(85)] _ Hydrodynamic limit transition for a quasi-one-dimensional system, Turán Memorial Lectures, Budapest, manuscript, 1985.

[S-Ch(85)] Ya. G. Sinai and N.I. Chernov, Ergodic properties of certain systems of 2- $D$ discs and 3-D balls, manuscript.

$[\mathrm{S}-\mathrm{Ch}(87)] \ldots$ Ergodic properties of certain systems of 2-D discs and 3-D balls, Russian Math. Surveys (3) 42, 181-207.

[Sz(95)] D. Szász, Boltzmann's ergodic hypothesis, a conjecture for centuries?, Studia Sci. Math. Hung, to appear.

[V(79)] L. N. Vaserstein, On systems of particles with finite range and/or repulsive interactions, Commun. Math. Phys. 69, 31-56.

Mathematical Institute of the Hungarian Academy of Sciences, H-1364, B UDAPEST, P. O. B. 127 , H UnGARY

E-mail address: nandor@math-inst.hu, szasz@math-inst.hu 